\section{Endocapsular iris reconstruction implants for acquired iris defects: a clinical study}

S Srinivasan ${ }^{1,2}$, C Yuen ${ }^{1,2}$, M Watts $^{1}$ and S Prasad ${ }^{1}$

\begin{abstract}
Purpose To report the clinical efficacy, safety, and long-term follow-up data on the use of endocapsular iris reconstruction implants (IRIs) during cataract surgery in patients with acquired iris defects.

Methods Single centre, retrospective, noncomparative, interventional case series. Five eyes of four patients with acquired iris defects and visually significant cataracts underwent clear cornea phacoemulsification and intraocular lens (IOL) implantation combined with insertion of endocapsular IRI. Data on the best-corrected visual acuity (BCVA), degree of preoperative and postoperative glare, photophobia, surgical complications, and longterm implant stability were analysed.

Results In all patients, IRI were successfully placed within the capsular bag during cataract surgery. There were no intraoperative or postoperative complications. Mean follow-up period was 29 months (range, 16-42). BCVA, subjective glare, and photophobia improved in all five eyes. Desired anatomic results were achieved in all of them.

Conclusions In patients with acquired iris defects, implantation of endocapsular IRI during cataract surgery appears to be a safe and effective procedure. At a mean time gate of 29 months, both IOLs and IRI appeared to remain stable within the capsular bag.

Eye (2007) 21, 1109-1113; doi:10.1038/sj.eye.6702472; published online 9 June 2006
\end{abstract}

Keywords: acquired iris defect; cataract surgery; iris reconstruction; iris implant

\section{Introduction}

An intact iris diaphragm is essential for accurate visual function as it decreases aberrations from the crystalline lens and increases the depth of focus. ${ }^{1}$ Symptoms of partial or total aniridia range from decreased vision and cosmetic concerns to incapacitating glare and photophobia. Various techniques to overcome visual function impairments arising from partial or total aniridia have been described, which include corneal tattooing, ${ }^{2,3}$ coloured contact lenses, and iridoplasty. ${ }^{4,5}$

In 1991 in conjunction with Sundmacher of the University of Düsseldorf, Morcher ${ }^{\circledR}$ (Stuttgart, Germany) developed a special intraocular lens (IOL) with an integrated iris diaphragm to correct congenital and traumatic forms of aniridia. These implants were CE (Conformité Européenne) certified in 1994 and have been available in Europe since then. Artificial iris implants are currently being manufactured by two European companies (Morcher, Stuttgart, Germany and Ophtec BV, Groningen, The Netherlands).

The first reported use of artificial iris implants were by Sundmacher et $a l^{6}$ and Reinhard et $\mathrm{al}^{7}$ in 1994, where they described using a black diaphragm single-piece IOL in congenital aniridia. Since then, there have been several reports on the use of artificial iris implants for congenital/acquired iris defects and congenital aniridia. ${ }^{8-12}$ Based on the extent of iris defect and presence or absence of capsular support, these implants can either be placed within the capsular bag, ciliary sulcus, or in the posterior chamber secured by scleral sutures. In cases of iris defects (partial or total) with coexisting cataracts, endocapsular aniridic rings (partial or total) can be combined with cataract surgery.

We report the clinical efficacy, safety, and long-term follow-up data on the use of endocapsular iris reconstruction implant (IRI) (Morcher, Stuttgart, Germany; Figure 1) in five eyes of four patients with acquired iris defects.
${ }^{1}$ Department of Ophthalmology, Arrowe Park Hospital, Wirral, UK

${ }^{2}$ St Paul's Eye Unit, Royal Liverpool University Hospital, Liverpool, UK

Correspondence: S Srinivasan, St Paul's Eye Unit, $8 Z$ Link,

Royal Liverpool University Hospital,

Prescot Street, Liverpool, Merseyside L7 8XP, UK Tel: + 44151706 2134; Fax: +44 1517065861 .

E-mail: sathish@tiscali.co.uk

Received: 14 March 2006 Accepted: 23 April 2006 Published online: 9 June 2006

Presented in part at American Academy of Ophthalmology Annual Meeting, October 2005, Chicago, Illinois.

The authors have no financial interest in any of the products or procedures listed in the article 


\section{Case reports}

\section{Case 1}

A 22-year-old male was referred with a history of blurred vision, glare, and photophobia in both eyes (BE). Ocular history was significant for bilateral congenital cataracts for which he had undergone bilateral temporal optical iridectomies as a child. Best-corrected visual acuities (BCVAs) were 6/18 in the right eye (RE) and 6/36 in the left eye (LE). He complained of incapacitating glare and photophobia in BE. Intraocular pressures were normal with open angles on gonioscopy. Biomicroscopy revealed bilateral temporal optical iridectomies extending 5 clock hours (from 0700 to 1100) in the RE and 4 clock hours (from 0200 to 0500) in the LE with bilateral lamellar cataracts (Figure 2a and b). Fundus examination was unremarkable. Following informed consent, he underwent phacoemulsification and IOL implantation combined with implantation of two IRI (type 50C, Morcher) in the RE. During the operation, before implantation, one of the fins in the 50C implants cracked while loading and had to be replaced with a readily available spare. At 6 weeks follow-up, he had an UCVA of 6/9 with marked reduction in glare and photophobia. A similar procedure was performed in the LE 6 months later. At last follow-up (34 months for RE and 28 months for LE), BCVAs were 6/6 BE with well-centred IOLs and well-aligned IRIs (Figure $2 \mathrm{c}$ and $\mathrm{d}$ ).

\section{Case 2}

A 76-year-old male was referred with decreasing vision, glare, photophobia, and dilated pupil in his RE. He had sustained blunt ocular trauma in the past, which resulted in a distorted and dilated pupil. On examination, BCVAs were 6/36 RE and 6/6 LE. Biomicroscopy showed a clear cornea, $7 \mathrm{~mm}$ fixed dilated pupil, and a cortical cataract. Gonioscopy showed no evidence of angle recession.

Fundus examination was unremarkable. Examination of the LE was normal. He underwent phacoemulsification

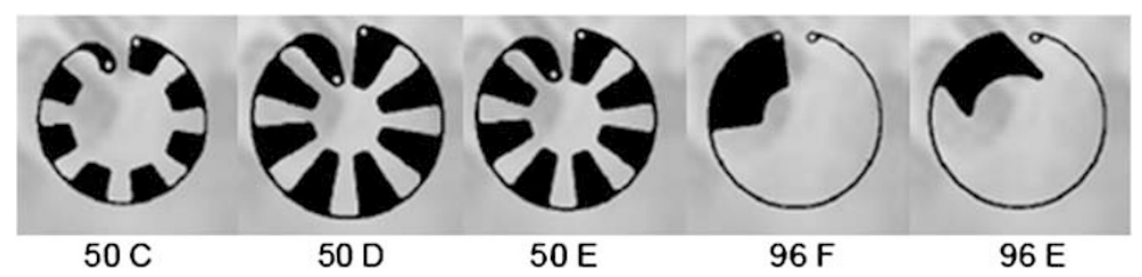

Figure 1 Types of currently available Morcher endocapsular IRIs.
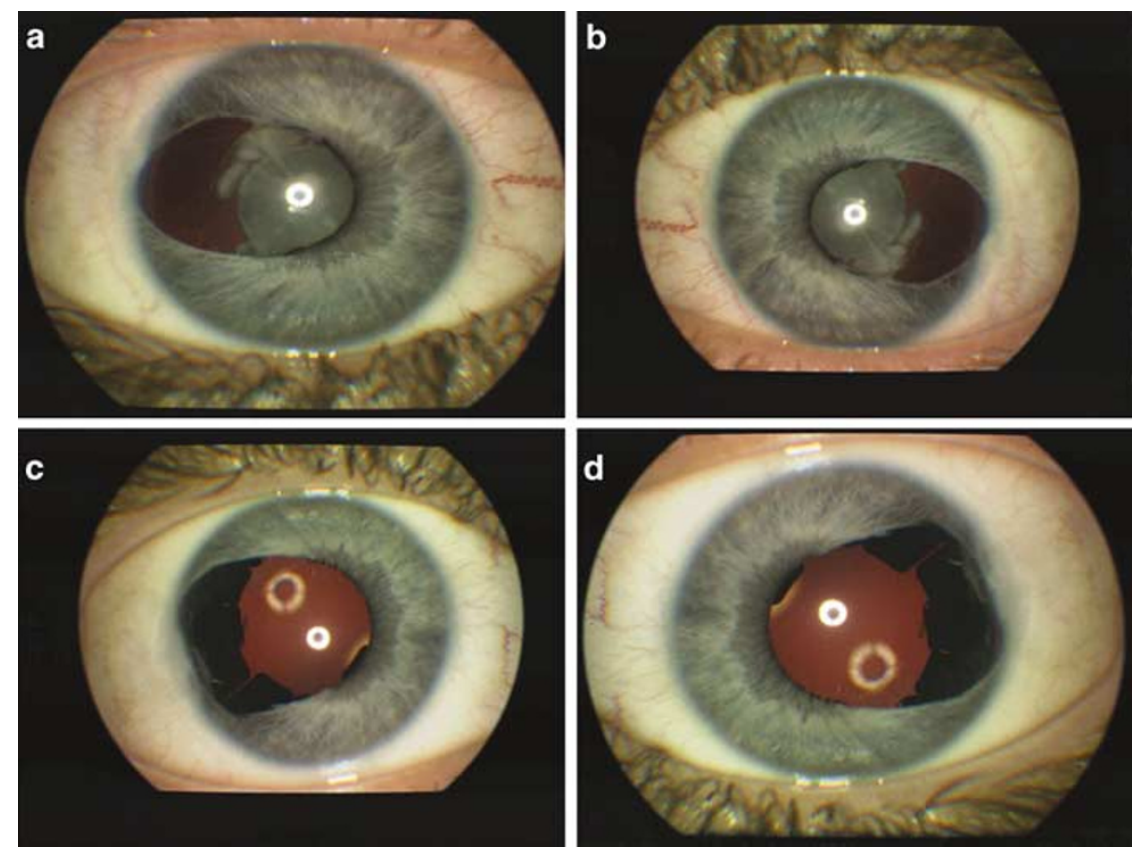

Figure 2 (a, b) Case 1, both eyes showing acquired sectoral temporal iris defects and congenital lamellar cataracts. (c, d) Postoperative photographs showing pseudophakia and well-centred type 50C endocapsular implants in both eyes. 
and IOL implantation combined with implantation of two IRIs (type 50C, Morcher) within the capsular bag (Figure 3a-f). There were no intraoperative complications. Postoperatively, he achieved a UCVA of $6 / 9$ at week 1 with significant reduction in glare and photophobia, and at 6 weeks, his BCVA improved to 6/6. At 16 months follow-up, he continues to have a centred IOL and a well-aligned IRI.

\section{Case 3}

A 69-year-old male sustained a penetrating injury in his RE in 2002. His cornea subsequently

decompensated for which he underwent a successful penetrating keratoplasty. After 2 years, he presented with increasing symptoms of monocular diplopia, which emanated from his superior traumatic iris defect (Figure 4). BCVAs were 6/60 RE and 6/9 LE. Biomicroscopy showed a clear corneal graft, 2 clock hours of superior iris defect, and nuclear sclerotic cataract in his RE. He underwent phacoemulsification combined with implantation of a type $96 \mathrm{~F}$ 'single-fin' IRI (Figure $5 \mathrm{a}-\mathrm{c}$ ). There were no intraoperative complications. Postoperatively, his BCVA improved to $6 / 9$ with no symptoms of monocular diplopia. At last follow-up (42 months postoperatively), he had a centred IOL within the bag, and a perfectly aligned IRI.
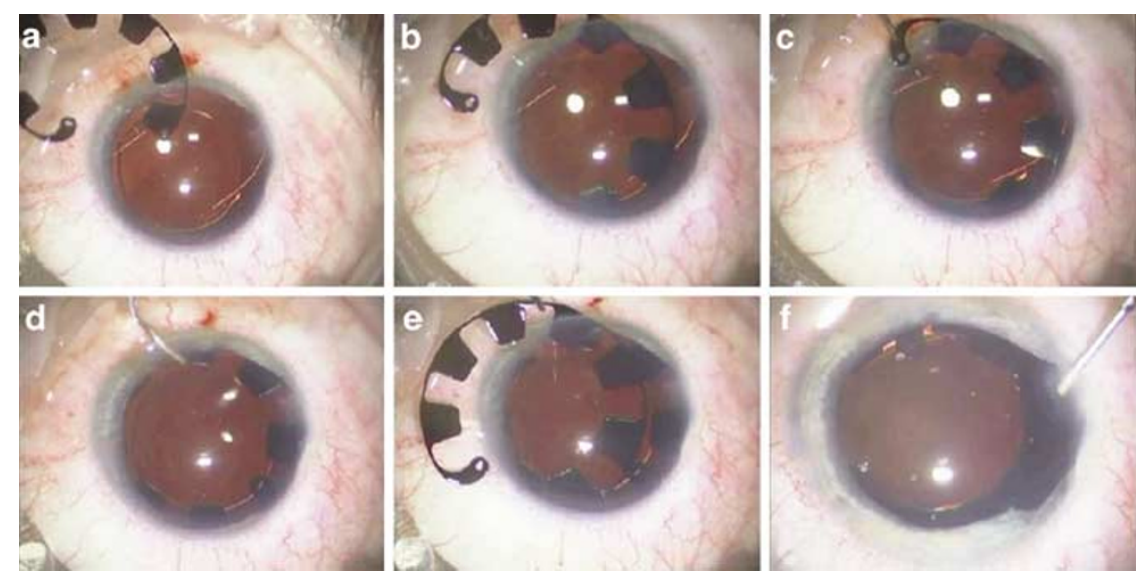

Figure 3 (a-f) Intraoperative photograph showing the insertion of two type 50C IRIs.

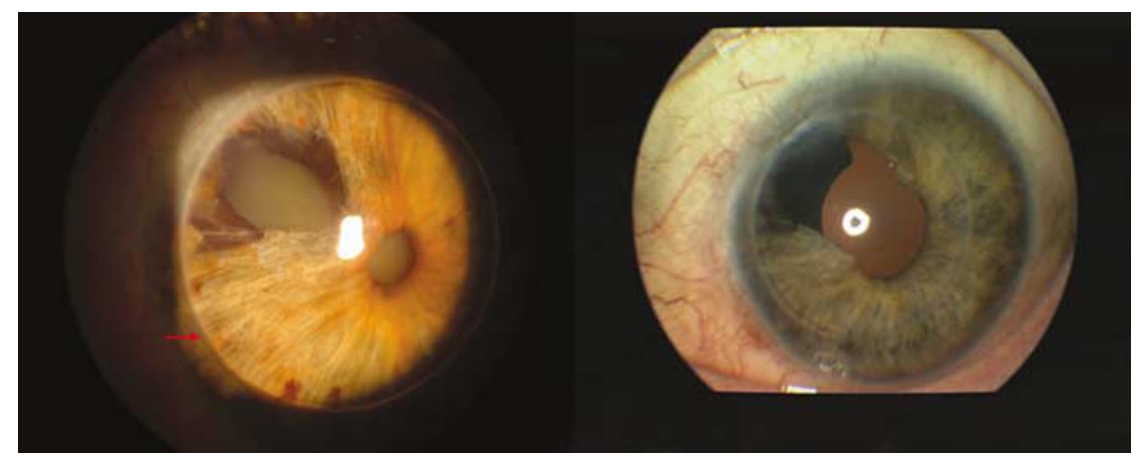

Figure 4 (Left) Case 3, RE, preoperative photograph showing a clear corneal graft (red arrow), superior acquired iris defect, and nuclear sclerotic cataract. (Right) Postoperative photograph showing pseudophakia and insertion of a type 96F single 'fin' IRI.
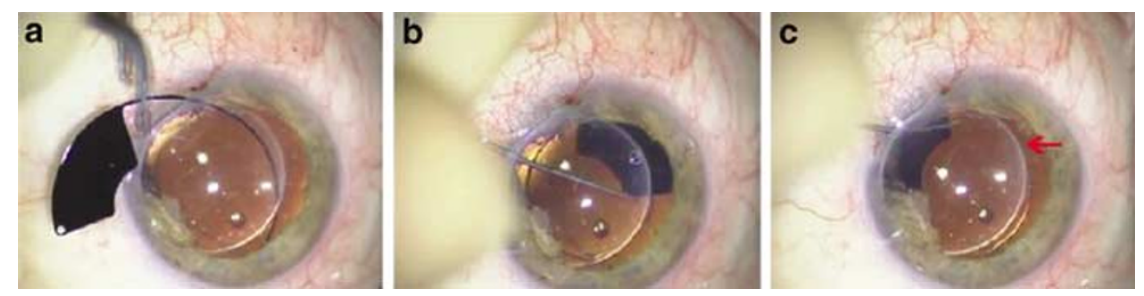

Figure 5 (a-c) Intraoperative photograph showing the steps involved in the insertion of type 96F single 'fin' IRI. 


\section{Case 4}

A 73-year-old male sustained ocular and orbital injuries to the RE following blunt trauma. He presented with orbital floor fractures, traumatic mydriasis ( $8 \mathrm{~mm}$ pupil in photopic conditions), and traumatic cataract with phacodonesis. He had a BCVA of 6/60 in the RE. Following repair of his orbital fractures, he underwent phacoemulsification and IOL implantation, combined with insertion of a $14 \mathrm{~mm}$ capsular tension ring (type 14A, Morcher) in order to stabilise the capsular bag and two type 50C endocapsular IRIs. There were no intraoperative complications. Postoperatively, he had a BCVA of $6 / 9$ in the RE with marked subjective improvement in his glare symptoms. At 25 months follow-up, he had a well-centred, stable IOL and a well-aligned IRI.

\section{Surgical procedure}

In all patients, following peribulbar anaesthesia, a superior $2.8 \mathrm{~mm}$ clear corneal wound was constructed. A cohesive ophthalmic viscosurgical device (OVD) (Healon, AMO, CA, USA) was used to maintain the anterior chamber. A $6 \mathrm{~mm}$ capsulorrhexis was fashioned. In one patient (BE of Case 1), Vision blue (Dorc, The Netherlands) was used to visualize the anterior capsule during capsulorrhexis. In one eye (Case 4), a capsular tension ring (type 14A, Morcher) was used to stabilize the capsular bag before phacoemulsification. Following endocapsular phacoemulsification, a multipiece silicone IOL (Soflex, Bausch and Lomb Surgical Inc., CA, USA) was implanted within the capsular bag. After implantation of the IOL, the cohesive OVD was replaced with a high-density OVD (Healon GV, AMO, Santa Ana, CA, USA) to 'open up' the potential space between the anterior capsule and the IOL within the bag. The corneal wound was enlarged to $3.5 \mathrm{~mm}$ and the preselected endocapsular IRI (either $50 \mathrm{C}$ or $96 \mathrm{~F}$ ) was dialled into the bag through the corneal wound. With the type 50C, two implants were sequentially placed within the bag and dialled in to position so that the fins interlocked to create a complete ring. With the type $96 \mathrm{~F}$, the implant was dialled within the bag until the single fin corresponded with the area of sectoral iris defect. At the end of the procedure, the OVD was aspirated and corneal stromal hydration was performed to create a self-sealing wound. A drop of chloramphenicol was placed in the inferior conjunctival sac. Postoperatively, all eyes were treated with topical steroids and antibiotics for 4 weeks.

\section{Discussion}

Partial or total loss of iris tissue can occur owing to several reasons. These include congenital absence, trauma, and iatrogenic causes. Several techniques are currently available to treat both the cosmetic and symptomatic aspects of aniridia. These range from the non-invasive option of occlusive coloured contact lenses to more invasive procedure like corneal tattooing or the technique of using IRIs. It was as early as 1964 when Choyce $^{13}$ reported the first use of anterior chamber IOLs with a coloured diaphragm in eyes with traumatic aniridia.

In 1994, Sundmacher et $a l^{6}$ and Reinhard et $a l^{7}$ independently reported the first use of a single-piece black iris diaphragm IOL in eyes with congenital aniridia during cataract surgery, but experienced problems during implantation, persistent intraocular inflammation, and secondary glaucoma. ${ }^{6}$ Since then, the IRIs have been modified ${ }^{12}$ and there are several types and models currently available depending on the location into which they are implanted. The choice for implantation is primarily dictated by the amount of residual iris tissue and the phakic status of the eye. In aphakic eyes with partial or total aniridia, a single-piece iris diaphragm IOL can either be placed in the anterior chamber supported by iris enclavation ${ }^{14}$ or in the absence of iris support, in the ciliary sulcus ${ }^{8,12,15}$ or suture fixated to the sclera (type 67G, Morcher). ${ }^{10,12,15}$ The main disadvantages of these implants are the need for a large incision, secondary glaucoma and chronic iritis., 9,12

In the presence of capsular support, endocapsular IRIs can be placed 'in the bag' during primary cataract surgery 9,16 or as a secondary procedure in a pseudophakic eye by viscodissection to reopen the capsular bag. ${ }^{9}$ Two different types of these endocapsular iris ring implants are currently available. Type 50 (C, D, and $\mathrm{E}$, Morcher) is used for complete reconstruction of iris in cases of total aniridia and in cases where sectoral loss of iris tissue extends more than 3 clock hours. These rings are made of black polymethylmethacrylate (PMMA) with an overall diameter of $10.75 \mathrm{~mm}$. Two of these rings are sequentially implanted within the bag through a small incision $(3.5 \mathrm{~mm})$ and dialled into position so that the 'fins' interlock against each other to produce a complete ring. Pupillary sizes of 6,4 , and $3.5 \mathrm{~mm}$ can be reconstructed by using the ring types $50 \mathrm{C}$, $\mathrm{D}$, and E, respectively. Partial iris defects extending less than 3 clock hours can be reconstructed by using the types 96 G, E, and F single 'fin' endocapsular iris implants. Sectoral iris loss between 3 and 6 clock hours can either be reconstructed by inserting two single fin rings ${ }^{9}$ or by using two 50C rings as demonstrated in Case 1. The endocapsular location of these implants avoids irritation to ciliary body and angle structures, thereby minimising postoperative inflammation. In this cohort, we encountered no more inflammation than one would expect following a routine phacoemulsification 
procedure. For the same reason, the incidence of secondary glaucoma becomes negligible. At a mean follow-up period of 29 months (range, 16-42), no eye in this cohort developed persistent intraocular inflammation or secondary glaucoma.

The main drawback of this implant is that they are brittle (caused by the pigment treatment of the PMMA material) and are susceptible to fracture during implantation. We encountered this problem during implantation of the type $50 \mathrm{C}$ implant in Case 1 . We recommend having a spare implant available in the operating room during such procedures. We believe that a large capsulorrhexis $(6 \mathrm{~mm})$ and an intact capsular bag is an essential prerequisite during implantation of the IRIs, as capsular phimosis (secondary to a small capsulorrhexis) and breach in the anterior capsular integrity can lead to decenteration and migration of the implant to the ciliary sulcus, respectively. ${ }^{16}$ None of the cases in these series had any intraoperative or late postoperative complications at a mean time gate of 29 months. No posterior capsular opacification has been noted to date.

In conclusion, implantation of endocapsular IRIs during cataract surgery appears to be a safe and effective technique in reducing glare disability and improving visual outcome.

\section{References}

1 Slomovits TL, Glaser JS. The pupils and accommodation. In Tasman W, Jaeger E (eds). Duane's Clinical Ophthalmology, Vol. 2, chapter 15. Lippincott Williams and Wilkins: Philadelphia, PA, 1998.

2 Burris TE, Holmes-Higgins DK, Silvestrini TA. Lamellar intrastromal corneal tattoo for treating iris defects (artificial iris). Cornea 1998; 17: 169-173.
3 Reed JW. Corneal tattooing to reduce glare in cases of traumatic iris loss. Cornea 1994; 13: 401-405.

4 Alger LG. The cause and treatment of poor vision in aniridia. Am J Ophthalmol 1945; 28: 730-735.

5 Hersh PS, Shingleton BJ, Kenyon KR. Anterior segment trauma. In: Albert DM, Jakobiec FA (ed). Principles and Practise of Ophthalmology. Saunders: Philadelphia, PA, 1994; 3383-3403.

6 Sundmacher R, Reinhard T, Althaus C. Black diaphragm intraocular lens in congenital aniridia. Ger J Ophthalmol 1994; 3: 197-201.

7 Reinhard, Sundamcher R, Althaus C. Irisblenden-IOL bei Traumatischer Aniridie (Iris black diaphragm intraocular lenses in traumatic aniridia). Klin Monatsbl Augenheilkd 1994; 205: 196-200.

8 Tanzer DJ, Smith RE. Black iris-diaphragm intraocular lens for aniridia and aphakia. J Cataract Refract Surg 1999; 25: 1548-1551.

9 Burke SE, Da Mata AP, Snyder ME, Cionni RJ, Cohen JS, Osher RH. Prosthetic iris implantation for congenital, traumatic, or functional iris deficiencies. J Cataract Refract Surg 2001; 27: 1732-1740.

10 Osher RH, Burk SE. Cataract surgery combined with implantation of an artificial iris. J Cataract Refract Surg 1999; 25: 1540-1547.

11 Mavrikakis I, Casey JMH. Phacoemulsification and endocapsular implantation of an artificial iris intraocular lens in traumatic cataract and aniridia. J Cataract Refract Surg 2002; 28: 1088-1090.

12 Reinhard T, Engelhardt S, Sundmacher R. Black diaphragm aniridia intraocular lens for congenital aniridia: long-term follow-up. J Cataract Refract Surg 2000; 26: 375-381.

13 Choyce P. Intra-Ocular Lenses and Implants, Vol. 27-32. HK Lewis: London, England, 1964, pp 162-178.

14 Hanumanthu S, Webb LA. Management of traumatic aniridia and aphakia with an iris reconstruction implant. J Cataract and Refract Surg 2003; 29: 1236-1238.

15 Thompson CG, Fawzy K, Bryce IG, Noble BA. Implantation of a black diaphragm intraocular lens for traumatic aniridia. J Cataract and Refract Surg 1999; 25: 808-813.

16 Mavrikakis I, Mavrikakis E, Syam PP, Bell J, Casey JH, Casswell AG. Surgical management of iris defects with prosthetic iris devises. Eye 2005; 19: 205-209. 